\title{
SJÖGREN'S SYNDROME AND NEUROMYELITIS OPTICA SPECTRUM DISORDER: CASE REPORT
}

Aurivan Essado Dantas ${ }^{1, \star}$, Matheus Fonseca Cardoso ${ }^{1}$, Ana Luisa Bagno de Almeida ${ }^{1}$, Larissa Maria Oliveira Gonzaga ${ }^{1}$, Camila Gabriela Xavier de Brito ${ }^{1}$, Samara de Quadros Lobê ${ }^{1}$, Victória Carneiro Dal Moro ${ }^{1}$, Leandro Augusto Tanure ${ }^{1}$

1.Universidade Federal de Minas Gerais, Belo Horizonte (MG), Brazil.

*Corresponding author: aurivan_ed@hotmail.com

\section{BACKGROUND:}

Neuromyelitis optica (NMO) is an inflammatory disease of the central nervous system characterized by the presence of detectable serum antibodies against the aquaporin-4 water channel (AQP4-lgG). AQP4-IgG-seropositive patients with limited or early presentations of NMO (including a first longitudinally extensive transverse myelitis attack or recurrent or bilateral optic neuritis) are included in the neuromyelitis optica spectrum disorders (NMOSD). These patients may have associated systemic diseases such as systemic lupus erythematosus or Sjögren's syndrome. Although this association is recognized, the mechanism and its clinical importance are still not fully understood. We present a case of an association between NMOSD and primary Sjögren's syndrome (pSS).

\section{CASE REPORT}

Male patient, 21 years old, with no history of previous diseases, with a report of subacute asymmetric lower limbs paraparesis, more intense on the left, with exalted osteotendinous reflexes, associated with urinary retention. Neuroaxis magnetic resonance imaging (MRI) showed extensive longitudinal myelitis, unchanged brain MRI and assessment of normal CSF. He was treated with intravenous methylprednisolone ( $1 \mathrm{~g} /$ day for 5 days) and maintained with oral prednisone $1 \mathrm{mg} / \mathrm{kg} /$ day. Approximately 1 month later the first event, the patient recurred with symptoms progression to the left upper limb and needed a new intravenous infusion of methylprednisolone. He also complained of xerophthalmia. Laboratory evaluation showed ANA 1:640 with dotted nuclear pattern, anti-Ro/SSA reagent in high titers, anti-AQP4 reagent 1:80, anti-SM nonreagent, protein electrophoresis with gamma globulin region distortion and biopsy of minor salivary glands evidencing chronic lymphocytic sialadenitis with a focus score of 1 (Fig. 1). Considering refractoriness, plasmapheresis and maintenance of immunosuppression with azathioprine was performed. The patient evolved with partial improvement of the symptoms, with strength gain and motor coordination, he continues in outpatient rehabilitation.

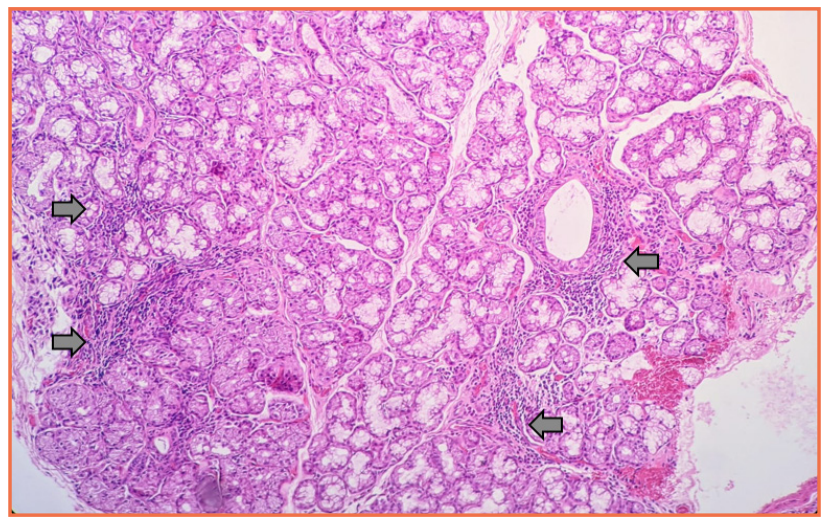

Figure 1. Minor salivary gland biopsy showing focal lymphocytic sialadenitis with focus score equal to 1 , represented by four focus (a cluster of 50 or more lymphocytes) in periductal or perivascular areas adjacent to normal acini in a $16 \mathrm{~mm}^{2}$ gland section.

\section{CONCLUSION}

In the clinical situation presented, we evaluated the infrequent association of pSS and NMOSD. It is noteworthy that the patient fulfills classification criteria for both (pSS-ACR/EULAR 2016 and NMOSD-AAN 2015). Recent evidence demonstrated that this association of diseases, especially when anti-AQP4 antibody is present, manifests itself in the general context of autoimmunity. Under these conditions, NMOSD does not appear to be a complication of pSS in the central nervous system. 